\title{
Re: More Extensive Lymph Node Dissection at Radical Prostatectomy is Associated with Improved Outcomes with Salvage Radiotherapy for Rising Prostate-specific Antigen After Surgery: A Long-term, Multi-institutional Analysis
}

Fossati N ${ }^{1}$, Parker $\mathrm{WP}^{2}$, Karnes $\mathrm{RJ}^{3}$, Colicchia $\mathrm{M}^{3}$, Bossi $\mathrm{A}^{4}$, Seisen $\mathrm{T}^{4}$, Di Muzio $\mathrm{N}^{5}$, Cozzarini $\mathrm{C}^{5}$, Noris Chiorda $\mathrm{B}^{5}$, Fiorino $\mathrm{C}^{5}$, Gandaglia $\mathrm{G}^{1}$, Bartkowiak $\mathrm{D}^{6}$, Wiegel $\mathrm{T}^{6}$, Shariat $\mathrm{S}^{7}$, Goldner $\mathrm{G}^{8}$, Battaglia $\mathrm{A}^{9}$, Joniau $\mathrm{S}^{9}$, Haustermans $\mathrm{K}^{10}$, De Meerleer $\mathrm{G}^{10}$, Fonteyne $\mathrm{V}^{11}$, Ost $\mathrm{P}^{11}$, Van Poppel $\mathrm{H}^{9}$, Montorsi $\mathrm{F}^{1}$, Briganti $\mathrm{A}^{1}$, Boorjian $\mathrm{SA}^{12}$

'San Raffaele Hospital, Clinic of Oncology, Unit of Urology, Milan, Italy

${ }^{2}$ University of Kansas Hospital, Department of Urology, Kansas City, Kansas, USA

${ }^{3}$ Mayo Clinic, Clinic of Urology, Rochester, Minnesota, USA

${ }^{4}$ Gustave Roussy Institute, Department of Radiation Oncology, Villejuif, France

${ }^{5}$ San Raffaele Hospital, Clinic of Radiotherapy, Milan, Italy

${ }^{6}$ University Hospital UIm, Department of Radiation Oncology, Ulm, Germany

${ }^{7}$ Medical University of Vienna, Department of Urology, Vienna, Austria

${ }^{8}$ Medical University of Vienna, Department of Radiation Oncology, Vienna, Austria

${ }^{9}$ University Hospitals Leuven, Department of Urology, Leuven, Belgium

${ }^{10}$ University Hospitals Leuven, Department of Radiotherapy, Leuven, Belgium

${ }^{11 G h e n t ~ U n i v e r s i t y ~ H o s p i t a l, ~ D e p a r t m e n t ~ o f ~ R a d i o t h e r a p y, ~ G h e n t, ~ B e l g i u m ~}$

${ }^{12}$ Mayo Clinic, Clinic of Urology, Rochester, Minnesota, USA

Eur Urol 2018;74:134-137. doi: 10.1016/j.eururo.2018.02.024.

\section{EDITORIAL COMMENT}

Residual, unresected lymph node disease has the potential to be a source for recurrence after salvage radiotherapy (sRT). The importance of pelvic lymph node dissection at the time of radical prostatectomy (RP) with regard to recurrence risk following sRT has not been previously determined. Therefore, the authors investigated whether the extent of nodal dissection at RP is associated with oncologic outcomes following SRT in men with increasing prostate-specific antigen (PSA) levels after surgery. They performed a multi-institutional review of men treated with sRT with a rising PSA after RP. The associations between lymph node yield and biochemical recurrence (BCR) as well as clinical recurrence (CR) after sRT were assessed. Seven hundred twenty eight patients were identified; of these, 221 were diagnosed with BCR and 116 with CR during a median follow-up of 8.4 years. The risk of BCR after sRT was inversely associated with the number of nodes resected, and increased extent of dissection was associated with a decreased risk of CR after sRT. The authors concluded that their data support the importance of an extensive lymph node dissection at surgery that may be used in prognosis assessment when sRT is being considered.

The authors should be congratulated for investigating an inventive notion which will pave the way for further work on the effects of surgical technique on treatments downstream in multi-modal approach to high-risk prostate cancer. However, the multi-institutional, retrospective design of the study cautions us to be watchful for inherent flaws such as non-standardized surgical as well as sRT technique, timing and indications for sRT, pathological processing and reporting, etc.

Özgür Yaycıoğlu, MD, FEBU

${ }^{\circ}$ Copyright 2018 by the Association of Urological Surgery / Journal of Urological Surgery published by Galenos Publishing House. 